\title{
Metachronous Basal Cell Carcinoma and Primary Plasmacytic Leukemia Associated With Dermatomyositis
}

\author{
Yuan YANG, Qibing XIE, Geng YIN \\ Department of Rheumatology and Immunology, West China Hospital of Sichuan University, Chengdu, China
}

\begin{abstract}
Dermatomyositis is associated with cancer frequently. However, successive occurrence of two malignancies in one person is rare. In this article, we present a 71-year-old female patient who was diagnosed as dermatomyositis based on skin lesions, muscle weakness, elevated muscle enzymes, electromyography, and muscle biopsy. Two years later, the patient had an ulcer on the left nasolabial groove. A resection was performed after basal cell carcinoma was confirmed by tissue biopsy. Two years after resection, serum $M$ protein, urinary $\lambda$ light chain, and neoplastic plasma cells infiltration in bone marrow biopsy of the patient were evaluated. Findings were compatible with the plasmacytic leukemia. Dermatomyositis patients may suffer from multiple tumors due to carcinogenic susceptibility. Therefore, physicians should continue to monitor dermatomyositis patients after the occurrence of the first tumor since they still have the possibility of developing another malignancy.

Keywords: Basal cell carcinoma; dermatomyositis; plasmacytic leukemia.
\end{abstract}

Dermatomyositis (DM) is an autoimmune inflammatory disease characterized by the association of progressive proximal muscle weakness and specific cutaneous signs, as well as multisystemic manifestations. ${ }^{1}$ Although the association between DM and elevated risks of comorbid cancers has been studied extensively, ${ }^{2}$ individuals with multiple malignancies associated with DM are rare. Some previous reports associated $\mathrm{DM}$ and two different types of malignancies in the same individual. ${ }^{3-5}$ To the best of our knowledge, this is the first case of the metachronous basal-cell carcinoma of the nasolabial fold and plasma cell leukemia occurring in the same DM patient over a period of time.

\section{CASE REPORT}

A 71-year-old female patient presented with a four-month history of muscle weakness, such as difficulty in raising her hands over the level of her shoulders and walking up and down the stairs. Physical examination revealed periorbital violaceous erythema and Gottron's papules on both elbows. Strength testing demonstrated a grade of 3/5 for the cervical muscle, upper and lower limbs. Laboratory tests showed elevated muscle enzymes with a creatine phosphokinase level of $2924 \mathrm{U} / \mathrm{L}$ (NR 20-140 U/L), alanine aminotransferase level of $186 \mathrm{U} / \mathrm{L}$ (NR <40 U/L), aspartate aminotransferase level of $151 \mathrm{U} / \mathrm{L}$ (NR $<35 \mathrm{U} / \mathrm{L}$ ), lactate dehydrogenase level of $834 \mathrm{U} / \mathrm{L}$ (NR 110-220 U/L), and hydroxybutyrate dehydrogenase level of 699 U/L (NR 72-182 U/L). Anti-nuclear antibody was positive at a titer of 1:1000, and anti-Jo-1 antibody was negative. Electromyography suggested myogenic damage. The muscle biopsy revealed a large number of inflammatory cells infiltrated along the vessels. Thus, the diagnosis of DM was established according to the Bohan and Peter's criteria. 6,7 The patient was initially treated with intravenous methylprednisolone $80 \mathrm{mg} / \mathrm{d}$ for seven days

\footnotetext{
Received: December 04, 2013 Accepted: January 16, 2014 Published online: November 12, 2014

Correspondence: Geng Yin, M.D. Department of Rheumatology and Immunology, West China Hospital of Sichuan University, 610000 Chengdu, China. Tel: +86-28-854 22393 e-mail: gengyin1975@gmail.com

(2014 Turkish League Against Rheumatism. All rights reserved.
} 


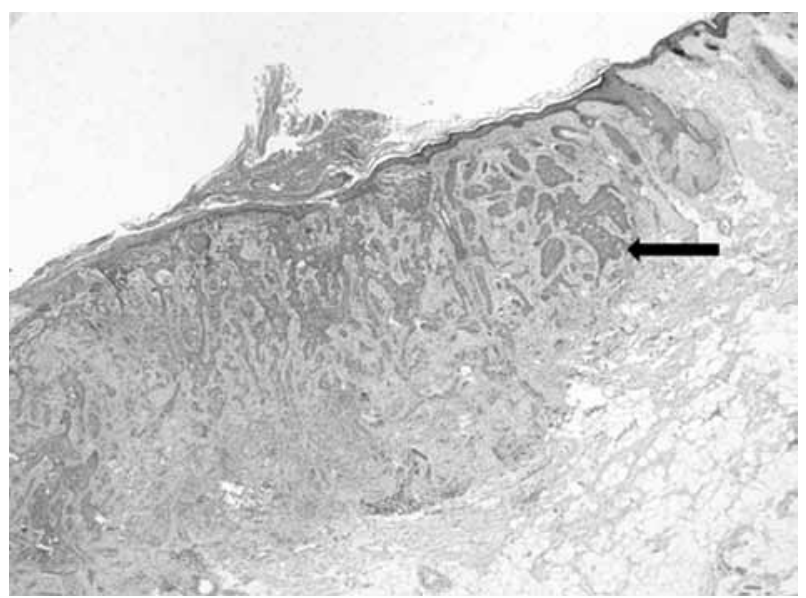

Figure 1. Skin biopsy reveals multiple nests of basal-cell carcinoma invading into the dermis (H-E x 10).

(then tapered gradually), followed by methotrexate $10 \mathrm{mg} /$ week, and hydroxychloroquine $200 \mathrm{mg}$ twice a day. The skin lesions resolved markedly and symptoms of muscle weakness relieved with treatment. At the time of discharge, she was kept on a maintenance oral dose of prednisone $7.5 \mathrm{mg}$ daily, and her symptoms were alleviated during the follow-up visit.

Two years later, the patient presented with an ulcer on the left nasolabial groove. Skin biopsy of the affected tissue revealed damaged epidermis with basal-cell carcinoma infiltration in the dermis (Figure 1), suggesting basal-cell carcinoma for which she underwent a resection. There were no clinical or laboratory signs suggestive of DM activity.

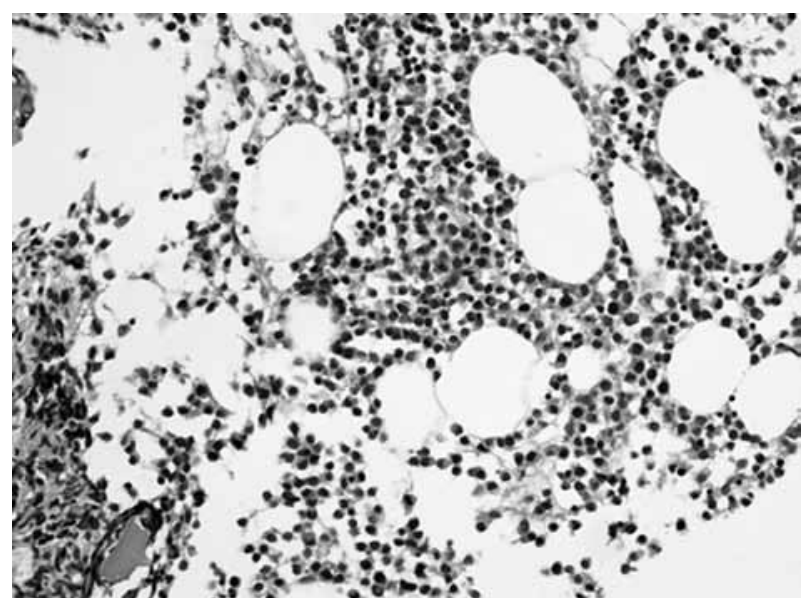

Figure 2. Bone marrow biopsy shows heavy infiltration by neoplastic plasma cells (H-E x 25).
However, two years after the diagnosis of basal-cell carcinoma, the patient was re-admitted to the hospital due to complaints of feebleness, palpitation and cough lasting for 10 days. Laboratory analysis showed an increased white blood cell count of $20 \times 10^{9} \mathrm{~L}$ (NR $4-10 \times 10^{9} / \mathrm{L}$ ), decreased red blood cell count of $1.28 \times 10^{12} / \mathrm{L}$ (NR $3.5-5.0 \times 10^{12} / \mathrm{L}$ ), and platelet count of $45 \times 10^{9} / \mathrm{L} \quad$ (NR 100-300 $\times 10^{9} / \mathrm{L}$ ). Serum electrophoresis revealed monoclonal protein of $68.24 \%$. The urinary lambda $(\lambda)$ light chain was 0.186 (NR <0.05). Bone marrow biopsy revealed diffuse fibrosis with collagen deposition, heavy infiltration of neoplastic plasma cells (CD138, PC positive), and $\lambda$ light chain restriction (Figure 2-4) with a total ratio of $66 \%$ for original and naive plasma cells. Hence, the diagnosis of $\lambda$ plasmacytic leukemia was confirmed. However, our patient rejected to undergo further evaluation and treatment due to personal reasons.

\section{DISCUSSION}

The association of neoplasms with DM is well documented. Dermatomyositis patients have a six to 10 folds increased possibility of developing cancer compared to the general population. ${ }^{8}$ In Europe, the most relevant malignancies include cancers in the ovary, lung, pancreas, stomach, and urinary bladder, as well as hematologic cancers including non-Hodgkin's lymphoma and Hodgkin's lymphoma, $, 8,9$ whereas breast,

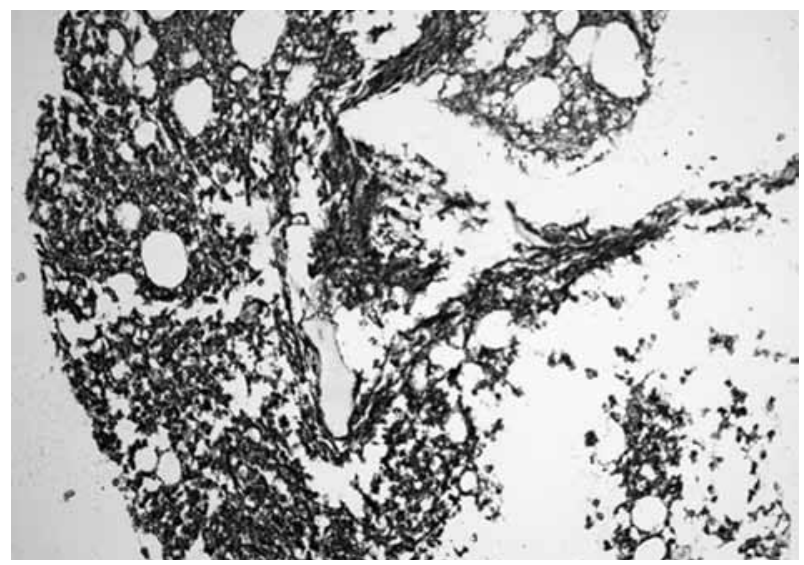

Figure 3. Immunohistochemistry shows infiltrated neoplastic cell expressing the plasmocyte labels, CD 138 (x 50). 


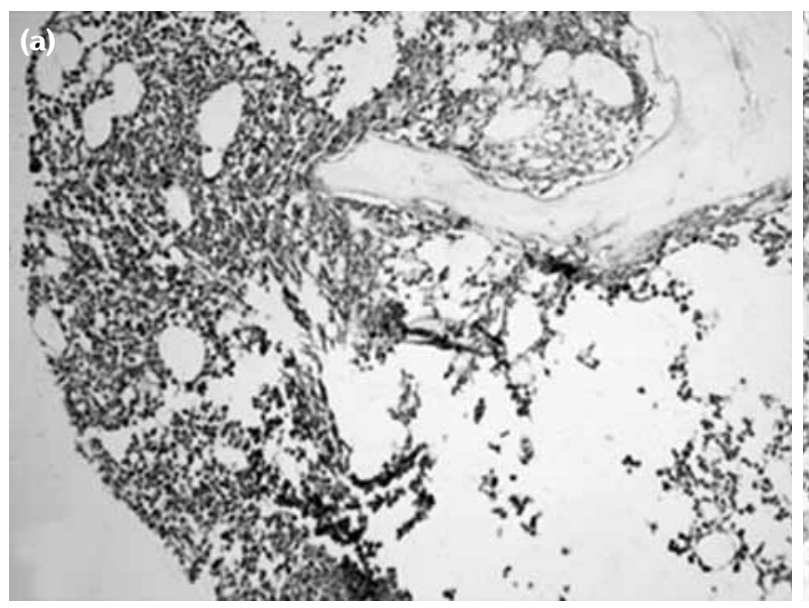

Figure 4. Immunohistochemistry shows lambda $(\lambda)$ light chain (b) expression (x 50).

stomach and nasopharyngeal cancers are most common in Asia. ${ }^{10,11}$ However, individuals with multiple malignancies associated with DM are rare. There have been some previous cases of DM associated with two different types of malignancies in the same individual. ${ }^{3-5}$ Of these, combination of breast and ovarian cancers is reported commonly. ${ }^{4,5}$ The literature shows that women with DM are more likely to suffer from multiple tumors than men. ${ }^{3-5}$ Some unrevealed relationship may exist between the incidence of cancer and the abnormal immune system function in the female reproductive system. However, the combination of the cutaneous covering and the hematological system is rare.

Based on the epidemiological observations, ${ }^{9}$ cancer-associated DM can be defined as a cancer occurring within three years from the diagnosis of DM. Studies commonly showed that cancerassociated DM is a paraneoplastic phenomenon. However, the etiology of DM in patients with cancer is poorly understood. It was demonstrated that antigenic myositis-specific autoantigens are expressed in both tumor cells and myoblasts, and then myositis-specific autoantigen is expressed in an initial tumor leading to the generation of both specific $\mathrm{T}$ and $\mathrm{B}$ cells targeting those antigens, and subsequently stimulate immune responses previously generated in the anti-tumor response..$^{12-15}$ These may explain the mechanism of the coexistence of DM and cancer.

Cancer risk in DM may be significantly high during the first year after the diagnosis of myositis. ${ }^{9}$ Successful anti-cancer treatments may provide better outcomes in cancer-associated DM. ${ }^{16}$ Thus, the determination of clinical and laboratory signs for association with malignancy are of great importance for the disease prognosis. In some studies, relatively high proportions of anti-155/140 antibodies were detected in cancer-associated DM patients, and these have an excellent positive predictive value $e^{12,13,17}$ Therefore, introduction of a commercial kit for this antibody to be applied in clinical screening may be beneficial. It was also shown that anti-Jo-1 antibody and cancer-associated DM, or interstitial lung disease and cancerassociated DM appear mutually exclusive. AntiJo-1 antibody and interstitial lung disease are both associated with a specific human leukocyte antigen class II haplotype, and anti-155/140 antibody is associated with human leukocyte antigen-DQA $1^{*} 0301,{ }^{17}$ suggesting that cancer risk in DM may be genetically determined. Thus DM patients may suffer multiple tumors due to genetic susceptibility. Therefore, we must closely follow-up any occurrence of other tumors by regular screening and long periods of follow-up after the development of the first tumor in DM patients. We should not ignore tumors from other systems such as skin and blood, even though most reported malignancies come from the female reproductive system.

\section{Declaration of conflicting interests}

The authors declared no conflicts of interest with respect to the authorship and/or publication of this article. 


\section{Funding}

The authors received no financial support for the research and/or authorship of this article.

\section{REFERENCES}

1. Huang YL, Chen YJ, Lin MW, Wu CY, Liu PC, Chen $\mathrm{TJ}$, et al. Malignancies associated with dermatomyositis and polymyositis in Taiwan: a nationwide populationbased study. Br J Dermatol 2009;161:854-60.

2. Bohan A, Peter JB, Bowman RL, Pearson CM. Computer-assisted analysis of 153 patients with polymyositis and dermatomyositis. Medicine (Baltimore) 1977;56:255-86.

3. Callen JP. Dermatomyositis and multiple malignancies in a patient treated with methotrexate. J Surg Oncol 1983;24:113-6.

4. Voravud N, Dimopoulos M, Hortobagyi G, Ross $M$, Theriault R. Breast cancer and second primary ovarian cancer in dermatomyositis. Gynecol Oncol 1991;43:286-90.

5. Murgic J, Prpic M, Kirac I, Camino-Varela AM, Bolanca A, Kusic Z. Dermatomyositis as paraneoplastic syndrome of peritoneal and ovarian relapse after long-term complete remission in patient with metastatic bilateral breast cancer. Coll Antropol 2012;36:325-9.

6. Bohan A, Peter JB. Polymyositis and dermatomyositis (first of two parts). N Engl J Med 1975;292:344-7.

7. Bohan A, Peter JB. Polymyositis and dermatomyositis (second of two parts). N Engl J Med 1975;292:403-7.

8. Hill CL, Zhang Y, Sigurgeirsson B, Pukkala E, Mellemkjaer L, Airio A, et al. Frequency of specific cancer types in dermatomyositis and polymyositis: a population-based study. Lancet 2001;357:96-100.

9. Chow WH, Gridley G, Mellemkjaer L, McLaughlin JK, Olsen JH, Fraumeni JF Jr. Cancer risk following polymyositis and dermatomyositis: a nationwide cohort study in Denmark. Cancer Causes Control 1995;6:9-13.

10. Lee SW, Jung SY, Park MC, Park YB, Lee SK. Malignancies in Korean patients with inflammatory myopathy. Yonsei Med J 2006;47:519-23.

11. Chen YJ, Wu CY, Shen JL. Predicting factors of malignancy in dermatomyositis and polymyositis: a case-control study. Br J Dermatol 2001;144:825-31.

12. Chinoy H, Fertig N, Oddis CV, Ollier WE, Cooper RG. The diagnostic utility of myositis autoantibody testing for predicting the risk of cancer-associated myositis. Ann Rheum Dis 2007;66:1345-9.

13. Kaji K, Fujimoto M, Hasegawa M, Kondo M, Saito Y, Komura K, et al. Identification of a novel autoantibody reactive with 155 and $140 \mathrm{kDa}$ nuclear proteins in patients with dermatomyositis: an association with malignancy. Rheumatology (Oxford) 2007;46:25-8.

14. Targoff IN, Mamyrova G, Trieu EP, Perurena O, Koneru B, O'Hanlon TP, et al. A novel autoantibody to a $155-\mathrm{kd}$ protein is associated with dermatomyositis. Arthritis Rheum 2006;54:3682-9.

15. Targoff IN. Myositis specific autoantibodies. Curr Rheumatol Rep 2006;8:196-203.

16. Madan V, Chinoy H, Griffiths CE, Cooper RG. Defining cancer risk in dermatomyositis. Part II. Assessing diagnostic usefulness of myositis serology. Clin Exp Dermatol 2009;34:561-5.

17. Targoff IN, Johnson AE, Miller FW. Antibody to signal recognition particle in polymyositis. Arthritis Rheum 1990;33:1361-70. 